\title{
A method for determining the emergence level of transformer technologies for green energy applications
}

\author{
Gaizka Garechana, Rosa Río-Belver, Enara Zarrabeitia, Izaskun Álvarez-Meaza
}

Department of business management, University of the Basque Country, Spain.

\begin{abstract}
Solid State Transformers (SST) are the result of merging the power electronics possibilities for voltage and frequency control with high-frequency transformers, and are expected to be a key component for enabling some important features that future energy grids must possess: reversibility, stability, modularity and compactness, among others. In addition to this, the possibilities of SSTs can be enhanced with advanced semiconductor materials such as Silicium Carbide (SiC), considerably improving the voltage and frequency ranges of this devices. This study aims at developing a quantitative method for characterizing the emergence level of SSTs and SiC-based transformers in three areas where these technologies can have a sizable impact: photovoltaic (PH) and eolic (EO) energy production and electric vehicle (EV) appliances. Results show that PH area will probably outpace the EO area in both technologies, but the attention of the scientific community may be shifting from PH in the SiC-based transformer technology. EV applications are, on average, closer to the life cycle's exponential growth stage than PH and EO areas, so it seems reasonable to expect a comparatively faster increase of both scientific and technology development activity in this field.
\end{abstract}

Keywords: SST; Silicium Carbide; Technology Forecasting; Emergent technologies. 


\section{Introduction}

Electrical transformers are devices that allow changing the voltage of electric current, a wellknown application of transformers is that of increasing the voltage of the alternating current (AC) generated in power stations from low to high (step-up transformer), in order to increase the voltage and reduce the current, given that less current means that less energy is lost when transporting said current to the customers. High voltage current, however, is dangerous for typical household purposes, so voltage must be reduced back to safe levels (step-down transformer) before its final use. The conventional transformer presents some drawbacks for its deployment in local, decentralized, renewable energy grids. First, conventional transformers are too big for many of these applications. Second, transformers are one-way tools, suited for energy distribution systems designed around big, centralized power plants, which comes in stark contrast with the operational aspects of the smart and clean technologies that are expected to substantially increase their share in the energy mix of the future (Roberts, 2018). There is a need for small and flexible transformation systems that can also deal with energy storage systems that work on direct current (DC).

Solid State Transformers (SST) are the result of merging the power electronics possibilities for voltage and frequency control with high-frequency transformers. One of the core tasks of power electronics in these devices is to increase the typical current frequency coming from the grid $(50 \mathrm{~Hz}$ in Europe) to a range between 10 and $20 \mathrm{KHz}$ in order to feed a highfrequency transformer that could be $20 \%$ smaller than a conventional transformer. This can be achieved using conventional silicon-based insulated-gate bipolar transistors (IGBT), at the expense of the reliability and limited handling of voltage $(6.5 \mathrm{Kv})$. New semiconductor materials such as the silicium carbide $(\mathrm{SiC})$ address these shortcomings, enabling SSTs to work at higher voltages and very high frequencies, thus achieving the maximum reduction in transformer size (Bhattacharya, 2017). The flexibility brought by the power electronics also allows the adjustment of the SST to frequent shifts in voltage and the requirements of a smart energy-management system (Abu-Siada, Budiri, \& Abdou, 2018).

The three-module approach proposed by Bhattacharya (2017) offers direct DC connection in the components of the SST, allowing to build direct interfaces with solar or other renewable energy technologies, thus avoiding extra DC - AC conversion steps and consequently, improving the efficiency. The possibilities of this multi-port structure go even further: Three module SiC-based SST systems can also be used to provide high voltage DC connection ports for electric vehicle (EV) quick chargers, using compact devices. The reversibility of the system could even allow using the local fleet of electric cars as a storage system for backing up the grid when necessary (Ronanki, Kelkar, \& Williamson, 2019). 


\section{Research goals}

The goal of the research presented in this paper is to develop a method suitable for determining the relative (since all the technologies studied in this paper are considered to be "emergent" according to the industry consensus) maturity of the applications of SST technology in the areas of photovoltaic energy (PH), eolic energy (EO) and the electric vehicle (EV), where the new transformer technologies are deemed to have a high impact.

The above mentioned method will also be applied to transformer technologies based on the advanced semiconductor material $\mathrm{SiC}$, in order to analyze the penetration of this semiconductor in the transformer industry, in the $\mathrm{PH}, \mathrm{EO}$ and $\mathrm{EV}$ areas.

\section{Methodology}

The basic premise underlying our methodology is that the emergence stage of the technology life cycle corresponds with the exponential growth stage of the logistic growth curve (Kucharavy \& De Guio, 2015) and consequently, the current degree of development and future prospectives of an emergent technology can be characterized by fitting the data corresponding to that technology to an exponential model.

\subsection{Data retrieval and subsetting}

The present study uses scientific publication and patent data for the characterization of the developments taking place in a technological field. According to the linear model of innovation (Godin, 2006), advances in scientific activity should come before the development efforts (patents) at the organizations. Data was retrieved by running the following queries on Scopus database (scientific publications):

- SST technology: SST: TITLE-ABS-KEY ( solid W/0 state W/0 transformer )

- $\mathrm{SiC}$ in transformer technology: ( TITLE-ABS-KEY ( "SILICON CARBIDE" OR "SILICIUM CARBIDE" OR carborundum OR sic ) AND TITLE-ABS-KEY ( transformer ) )

....and their approximate equivalents in Patseer (patents) database:

- SST technology: (TACD:(SOLID wd0 STATE WD0 TRANSFORMER))

- $\mathrm{SiC}$ in transformer technology: ((TACD:(("silicon carbide" OR "silicium carbide" OR "carborundum") AND TRANSFORMER) AND AC:(H02M*)))

In order to accomplish the goals stated in section 0, data was subsetted by applying text mining techniques to the "description" field of patents and "title, abstract \& keywords" fields of scientific publications. We assume that the presence of terms unequivocally associated with $\mathrm{PH}, \mathrm{EO}$ or EV applications in these fields is an evidence that can be used for subsetting 
our data in three subsamples corresponding to the aforementioned areas. This approach provides a total amount of twelve subsamples to perform our study: six subsamples corresponding to scientific activity (PH, EO, EV - Scopus) in both SST and SiC transformer technologies, and their equivalent in patenting activity, (PH, EO, EV - Patseer) in both SST and $\mathrm{SiC}$ transformer technology.

These being emergent technologies, we obtained very few data prior to year 2000, so the time interval was defined from 2003 to 2017 for $\mathrm{SiC}$ transformer data, and from 2011/12 to 2018 for SST data. We pragmatically selected the start of the interval by selecting the first year in which at least one record was obtained for two out of the three areas under analysis (PH, EO, $\mathrm{EV}$ ), for both SST and SiC transformer technologies.

\subsection{Fitting the data to the exponential curve}

The number of publications and patents corresponding to the twelve subsamples is fitted to an exponential model, according to the following method:

First, we parametrize the equation $y=a x^{b}$ by taking $\operatorname{logs} \log (y)=\log (a)+b * \log (x)$ and fitting a linear regression model to the log-transformed data. This will provide an initial estimation of $a$ and $b$ parameters that will be subsequently recalculated by using the nonlinear weighted least-squares (NLS) method on the data, as explained in Hastie (2017), using R. The total amount of patents and publications corresponding to each area (PH, EO, EV) will inform abut the diffusion achieved by the technologies under study (SST and SiC based transformers) while the parameter $b$ will inform about the relative maturity of said technologies in each area, considering that the higher the $b$, the higher are the expectations about the success of the technology in a particular area, and the closer is that technology to the exponential growth phase of the technology life cycle.

\section{Results}

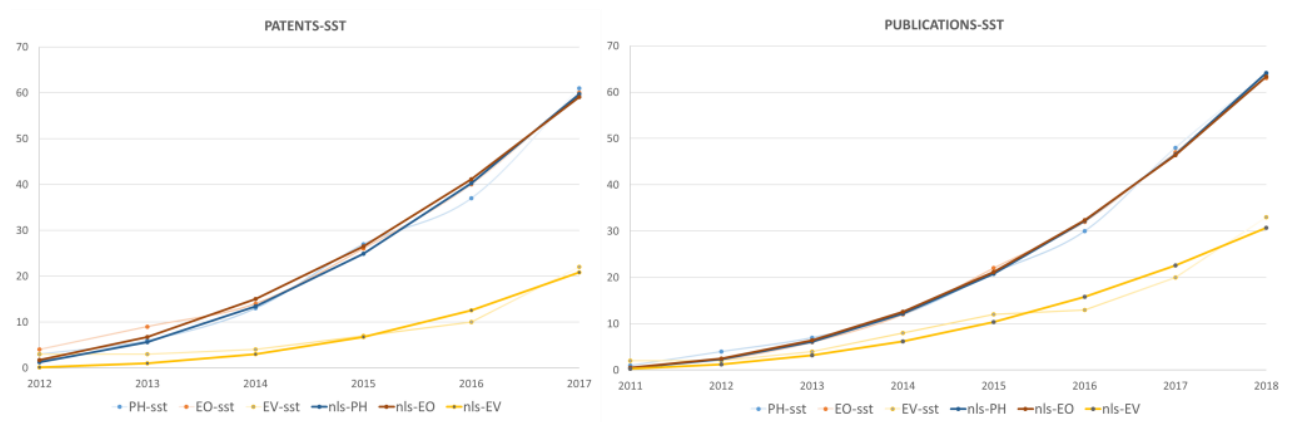

Figure 1 shows the accumulated patent and publication data corresponding to SST technology, as well as the results of fitting the model to the data: 

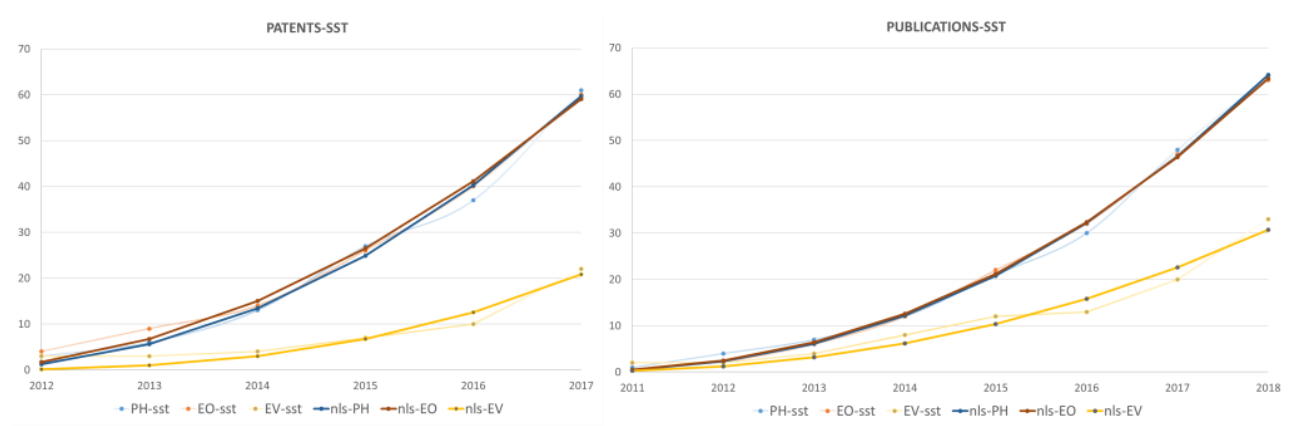

Figure 1. Data corresponding to SST technology (accumulated publications and patents). Colors indicate the application area $(\mathrm{PH}, \mathrm{EO}, \mathrm{EV})$ and the thickness of the line indicates whether it shows raw data (thin) or the result of fitting the NLS model (thick).

The first thing we notice is that both data sources (publications and patents) show approximately the same starting point for the data, according to the criteria we exposed on section 0 , and the patterns shown by data are also similar for both sources. The applications of SST to the EV area are fewer in number, and start to show a growth pattern later, when compared with $\mathrm{PH}$ and EO data. However, the $b$ parameter is significantly higher for patent data in EV area, as can be seen in the results presented in Table 1.

Figure 2 shows the results corresponding to the $\mathrm{SiC}$ transformer technology:
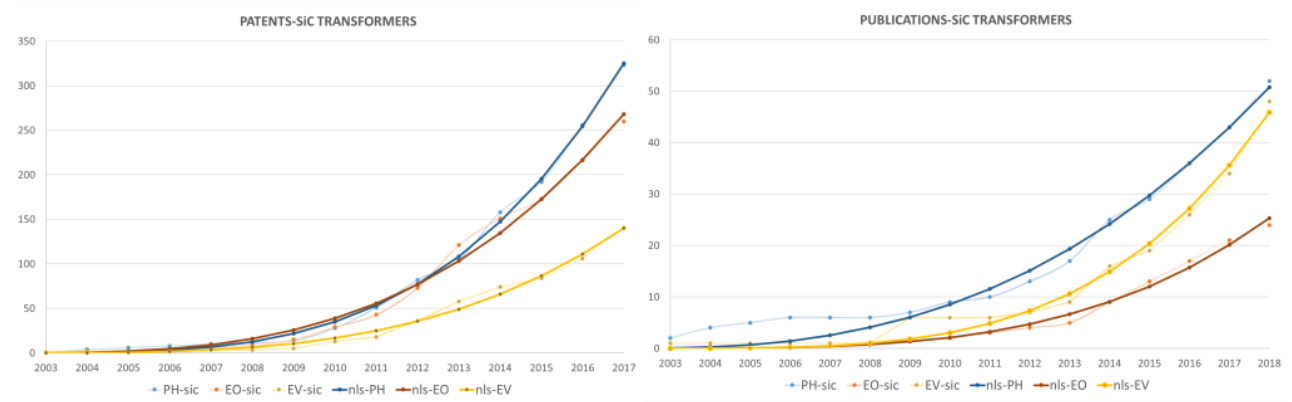

Figure 2. Data corresponding to SiC transformer technology(accumulated publications and patents). Colors indicate the application area $(P H, E O, E V)$ and the thickness of the line indicates whether it shows raw data (thin) or the result of fitting the NLS model (thick).

We observe the same phenomenon regarding the starting point of time intervals: both patent and publication data corresponding to this technology show the same starting point, according to our methodology. In this case, however, the pattern shown by the areas under analysis is significantly different for each data source. While the EV applications remain below $\mathrm{PH}$ and EO in patent data, research in EV applications is clearly outpacing EO and 
shows signs of surpassing the $\mathrm{PH}$ area, as can be seen in the $b$ parameter values shown in Table 1. EO and EV areas seem to be catching the attention of the scientific community at a fast pace when compared with PH, by looking at their $b$ values. This was not the case in SST technology. However, $\mathrm{PH}$ applications of $\mathrm{SiC}$ based-transformers are the most frequent, according to our data.

Table 1 shows the values of $b$ for the NLS models built for each technology, area and data source:

Table 1. Values of $b$ for each technology, area and data source.

\begin{tabular}{ccc}
\hline Technology - Area & Data source & $\boldsymbol{b}$ parameter \\
\hline SST-PH & Development (patents) & 2.15 \\
SST-EO & Development (patents) & 1.97 \\
SST-EV & Development (patents) & 2.79 \\
SST-PH & Research (scientific pub.) & 2.39 \\
SST-EO & Research (scientific pub.) & 2.33 \\
SST-EV & Research (scientific pub.) & 2.31 \\
SIC-PH & Development (patents) & 3.54 \\
SIC-EO & Development (patents) & 3.07 \\
SIC-EV & Development (patents) & 3.38 \\
SIC-PH & Research (scientific pub.) & 2.57 \\
SIC-EO & Research (scientific pub.) & 3.58 \\
SIC-EV & Research (scientific pub.) & 3.91 \\
\hline
\end{tabular}

The results show that EV applications have the highest average $b$ coefficient (3.09), while PH and EO applications show similar $b$ values (2.66 and 2.73, respectively).

\section{Discussion and conclusions}

A remarkable conclusion of this study is that the ideas of the linear model of innovation (Godin, 2006) fail to describe the behavior of SST and SiC transformer technologies: both research and development seem to be taking place simultaneously, according to our data. Research in these technologies is eminently applied science, where the boundaries between science and development become more porous (Kline, 1985), this could be an explanation of 
the phenomenon we have observed, but for our purposes, this trait of the technologies hinders the detection of early signs of emergence coming from the scientific world.

A head to head comparation between the two renewable energy sources under study suggests that the PH area will probably outpace the EO area in both technologies (SST and SiC-based transformers). The trends in scientific research in SiC-based transformers, however, suggest that the attention of the scientific community might be shifting from $\mathrm{PH}$ in this technology.

Perhaps the most interesting pattern can be found in the EV applications of both SST and SiC transformers. Data corresponding to EV area persistently shows a smaller yearly amount of research/patenting activity taking place when compared with the rest of the areas (with a single exception) but at the same time the higher average $b$ parameter is found in this area. According to our approach, this suggests that EV applications might be closer to the life cycle's exponential growth stage than $\mathrm{PH}$ and EO areas, so it seems reasonable to expect a comparatively faster development of both scientific and development activity in this field, when compared with $\mathrm{PH}$ and EO. This conclusion is reinforced, from our point of view, by the data presented in Figure 2 (right), which points at both a strong presence and exponential growth pattern in the academic activity related to $\mathrm{SiC}$ transformer applications in $\mathrm{EV}$ area. Considering that a substantial amount of research related with new semiconductor materials falls into the realm of basic science, technology forecasting efforts in this area should probably keep an eye on this sample of data, in order to look for early signals of emergence.

We hope that the results and the conclusions presented in this study will be useful for decision making in the field of renewable energies and the technologies related to electric vehicles, particularly for those professionals involved in technology forecasting practices.

\section{References}

Abu-Siada, A., Budiri, J., \& Abdou, A. (2018). Solid State Transformers Topologies, Controllers, and Applications: State-of-the-Art Literature Review. Electronics, 7(11), 298. https://doi.org/10.3390/electronics7110298

Bhattacharya, S. (2017). Smart Transformers Will Make the Grid Cleaner and More Flexible. Retrieved January 21, 2020, from https://spectrum.ieee.org/energy/renewables/smarttransformers-will-make-the-grid-cleaner-and-more-flexible

Godin, B. (2006). The Linear Model of Innovation: The Historical Construction of an Analytical Framework. Science, Technology \& Human Values, 31(6), 639-667. https://doi.org/10.1177/0162243906291865

Hastie, T. J. (2017). Statistical models in S. (T. J. Hastie \& J. M. Chambers, Eds.). Routledge.

Kline, S. (1985). Innovation is not a linear process. Research Management. Retrieved from http://www.ec.unipg.it/ez_new/index.php/ita/content/download/7711/35914/file/FILE $3 \_$Kline_ Innovation is not a linear process.pdf 
Kucharavy, D., \& De Guio, R. (2015). Application of Logistic Growth Curve. Procedia Engineering, 131, 280-290. https://doi.org/10.1016/J.PROENG.2015.12.390

Roberts, D. (2018). Renewable energy threatens to overwhelm the grid. Here's how it can adapt. Retrieved January 21, 2020, from https://www.vox.com/energy-andenvironment/2018/11/30/17868620/renewable-energy-power-grid-architecture

Ronanki, D., Kelkar, A., \& Williamson, S. S. (2019). Extreme Fast Charging TechnologyProspects to Enhance Sustainable Electric Transportation. Energies, 12(19), 3721. https://doi.org/10.3390/en12193721 